\title{
Latrophilins and Teneurins in Invertebrates: No Love for Each Other?
}

\author{
Torsten Schöneberg and Simone Prömel* \\ Medical Faculty, Rudolf Schönheimer Institute of Biochemistry, Leipzig University, Leipzig, Germany
}

\section{OPEN ACCESS}

Edited by:

Antony Jr Boucard, Centro de Investigación y de Estudios Avanzados (CINVESTAV), Mexico

Reviewed by:

Theodoros Tsetsenis, University of Pennsylvania,

United States

Konark Mukherjee, Fralin Biomedical Research Institute (FBRI), United States

*Correspondence:

Simone Prömel simone.proeme/@medizin.unileipzig.de

Specialty section: This article was submitted to Neuroendocrine Science, a section of the journal Frontiers in Neuroscience

Received: 08 January 2019

Accepted: 11 February 2019

Published: 12 March 2019

Citation:

Schöneberg $T$ and Prömel $S$ (2019) Latrophilins and Teneurins in Invertebrates: No Love for Each Other? Front. Neurosci. 13:154. doi: 10.3389/fnins.2019.00154
Transsynaptic connections enabling cell-cell adhesion and cellular communication are a vital part of synapse formation, maintenance and function. A recently discovered interaction between the Adhesion GPCRs Latrophilins and the type II single transmembrane proteins Teneurins at mammalian synapses is vital for synapse formation and dendrite branching. While the understanding of the effects and the molecular interplay of this Latrophilin-Teneurin partnership is not entirely understood, its significance is highlighted by behavioral and neurological phenotypes in various animal models. As both groups of molecules, Latrophilins and Teneurins, are generally highly conserved, have overlapping expression and often similar functions across phyla, it can be speculated that this interaction, which has been proven essential in mammalian systems, also occurs in invertebrates to control shaping of synapses. Knowledge of the generality of this interaction is especially of interest due to its possible involvement in neuropathologies. Further, several invertebrates serve as model organisms for addressing various neurobiological research questions. So far, an interaction of Latrophilins and Teneurins has not been observed in invertebrates, but our knowledge on both groups of molecules is by far not complete. In this review, we give an overview on existing experimental evidence arguing for as well as against a potential Latrophilin-Teneurin interaction beyond mammals. By combining these insights with evolutionary aspects on each of the interaction partners we provide and discuss a comprehensive picture on the functions of both molecules in invertebrates and the likeliness of an evolutionary conservation of their interaction.

Keywords: adhesion GPCRs, Latrophilins, Teneurins, invertebrates, interaction

\section{LATOPHILINS AND TENEURINS FORM A TRANSSYNAPTIC COMPLEX IN MAMMALS}

The formation of synapses is one of the key steps in warranting the development of a functioning neuronal network. This highly complex process is not fully understood, but it involves various interactions of molecules with adhesive and transmembrane signaling properties. A pair of proteins which has recently taken the stage to be essential for synaptic organization in many vertebrates are Latrophilins and Teneurins. Both have already been separately recognized as synaptic cell surface proteins several decades ago. 
Teneurins are large type II one-transmembrane domain proteins with a cytoplasmic $\mathrm{N}$-terminus and an extracellularly located C-terminus containing tyrosine-aspartate (YD) repeats and numerous epidermal growth factor (EGF) domains (Oohashi et al., 1999; Tucker and Chiquet-Ehrismann, 2006; Figure 1A). They have various neuronal functions, for example in mediating interneuronal connections, promoting synapse formation and shaping dendritic morphology in diverse types of neurons in vertebrates and invertebrates (Hong et al., 2012; Mosca et al., 2012; Antinucci et al., 2013; Berns et al., 2018). Consistently, the four vertebrate homologs (TEN1-4) are widely expressed in the developing and the adult brain, for instance in the hippocampus, the cerebellum and the visual cortex (Oohashi et al., 1999; Tucker et al., 2000; Rubin et al., 2002; Zhou et al., 2003; Kenzelmann et al., 2008). Studies on animal models further reveal the essential impact of Teneurins on neuronal circuits. For example, mice knockout for Ten3 display neurological defects, in particular deficits in visually mediated behavior (Leamey et al., 2007). Similarly, in zebrafish, knockdown of Ten-3 leads to retinal ganglion cell stratification defects (Antinucci et al., 2013).

The molecular details underlying Teneurin function involve the formation of homotypic or heterotypic dimers depending on the synapse type [summarized in Mosca (2015)]. Most details on Teneurin function, however, have not been collected in vertebrates, but using the fruit fly Drosophila melanogaster as a model (section "Latrophilins and Teneurins in D. melanogasterNo Evidence for Interaction").

The functions of Latrophilins have by far not been as well characterized as the ones of Teneurins. Latrophilins belong to the class of Adhesion G protein-coupled receptors (Adhesion GPCRs, aGPCRs). The three mammalian homologs (LPHN1-3/ADGRL1-3) comprise an intracellular C-terminus, a seven transmembrane region (7TM) and an extracellular $\mathrm{N}$-terminus containing a rhamnose-binding lectin (RBL), an olfactomedin (OLF), a hormone binding (HRM) and a GPCR autoproteolysis-inducing (GAIN) domain, which harbors the GPCR proteolytic site (GPS) (Figure 1B). Latrophilins first came into the focus of science as targets of $\alpha$-Latrotoxin, a component of the Black widow spider's toxin (Krasnoperov et al., 1996; Lelianova et al., 1997; Sugita et al., 1998). Specifically, Latrophilin-1 (LPHN1/ADGRL1) has subsequently been characterized to be expressed in various neurons of the murine central nervous system and evidence exists that LPHN1 is localized presynaptically (Silva et al., 2011; Vysokov et al., 2018) as well as on the post-synapse (Tobaben et al., 2000; Anderson et al., 2017). The impact of this localization on both sides of the synapse has not been clarified to date. Studies on LPHN3 in mouse and zebrafish models suggest a role for the receptor in the dopaminergic system and an association of variants in the receptor gene with the pathogenesis of attention-deficient hyperactive disorder (ADHD) (Arcos-Burgos et al., 2010; Lange et al., 2012; Wallis et al., 2012).

Teneurins and Latrophilins are both found enriched in neuronal growth cones (Nozumi et al., 2009). Recently, strong evidence has been provided that mammalian Teneurins and Latrophilins form heterophilic dimers at the synapse.
This interaction, which occurs between LPHN1 and the Teneurin homolog TEN2 [also termed Lasso (Silva et al., 2011)], is transsynaptic and mediates cell adhesion (Silva et al., 2011; Boucard et al., 2014; Li et al., 2018). As a consequence, it induces synapse formation in murine hippocampal neurons and neuronal cultures (Silva et al., 2011; Figure 2A). Another study has shown that besides TEN2 also TEN4, but not TEN1 is able to bind LPHN1 (Boucard et al., 2014). It needs to be noted that not for all Teneurin functions in neurons, interaction with Latrophilin is essential [reviewed in Mosca (2015)]. It has only proven vital for cell adhesion and synapse formation so far. For its other roles homophilic interactions or different heterophilic partners have been shown.

Although the molecular details of the interaction between Latrophilins and Teneurins have not been clarified yet, the regions within both molecules taking part in the intermolecular interaction have been roughly identified (Figure 2A) using binding assays and mutation analyses. For TEN2, the interaction is mediated via its C-terminal portion, mainly by a sequence within the Tox-GHH domain, the so-called Teneurin C-terminal-associated peptide (TCAP). This sequence can act as a bioactive peptide upon cleavage and shapes dendritic morphology, stimulates neurite outgrowth and mediates anxiety behavior (Wang et al., 2005; Al Chawaf et al., 2007a,b; Tan et al., 2011). Interestingly, besides this core sequence within the Tox-GHH domain, a 7-amino acid-long region located in a $\beta$-propeller close to the NHL (NCL-1/HT2A/Lin-41) repeats also regulates binding (Li et al., 2018). The same seems to be true for the interaction site within Latrophilins. While the presence of the RBL domain is mainly responsible for binding Teneurins (Boucard et al., 2014), an alternative exon encoding a region between RBL and OLF domains modulates binding affinity to TEN2 (Boucard et al., 2014). It needs to be noted that currently existing data on the partnership of Latrophilins and Teneurins does not exclude the possibility that the interaction occurs in the context of a larger complex involving other molecules. This scenario has been already proposed (Woelfle et al., 2015, 2016) based on the findings that Teneurin also interact with dystroglycans (Chand et al., 2012) and Latrophilins bind to Neurexins (Boucard et al., 2012) or (in a complex) to fibronectin leucine-rich transmembrane (FLRT) proteins (O'Sullivan et al., 2012, 2014; Jackson et al., 2015; Lu et al., 2015). These interaction partners are all expressed by neurons.

As both, Latrophilins and Teneurins, can act as ligand, it is conceivable that each of them functions as receptor transducing signals into their host cell. It has not been determined beyond doubt to date which of them is the ligand and which the signalreceiving molecule or if both of them signal. However, some studies show that Teneurins are cleaved at several distinct sites rendering liberated fragments (Wang et al., 2005), which are involved in different functions in the brain such as neurite outgrowth (Al Chawaf et al., 2007a; Erb et al., 2014). It has been suggested that one of these TEN2 fragments, generated by regulated proteolysis, is soluble and can still bind LPHN1 and trigger signaling (Silva et al., 2011; Vysokov et al., 2016, 


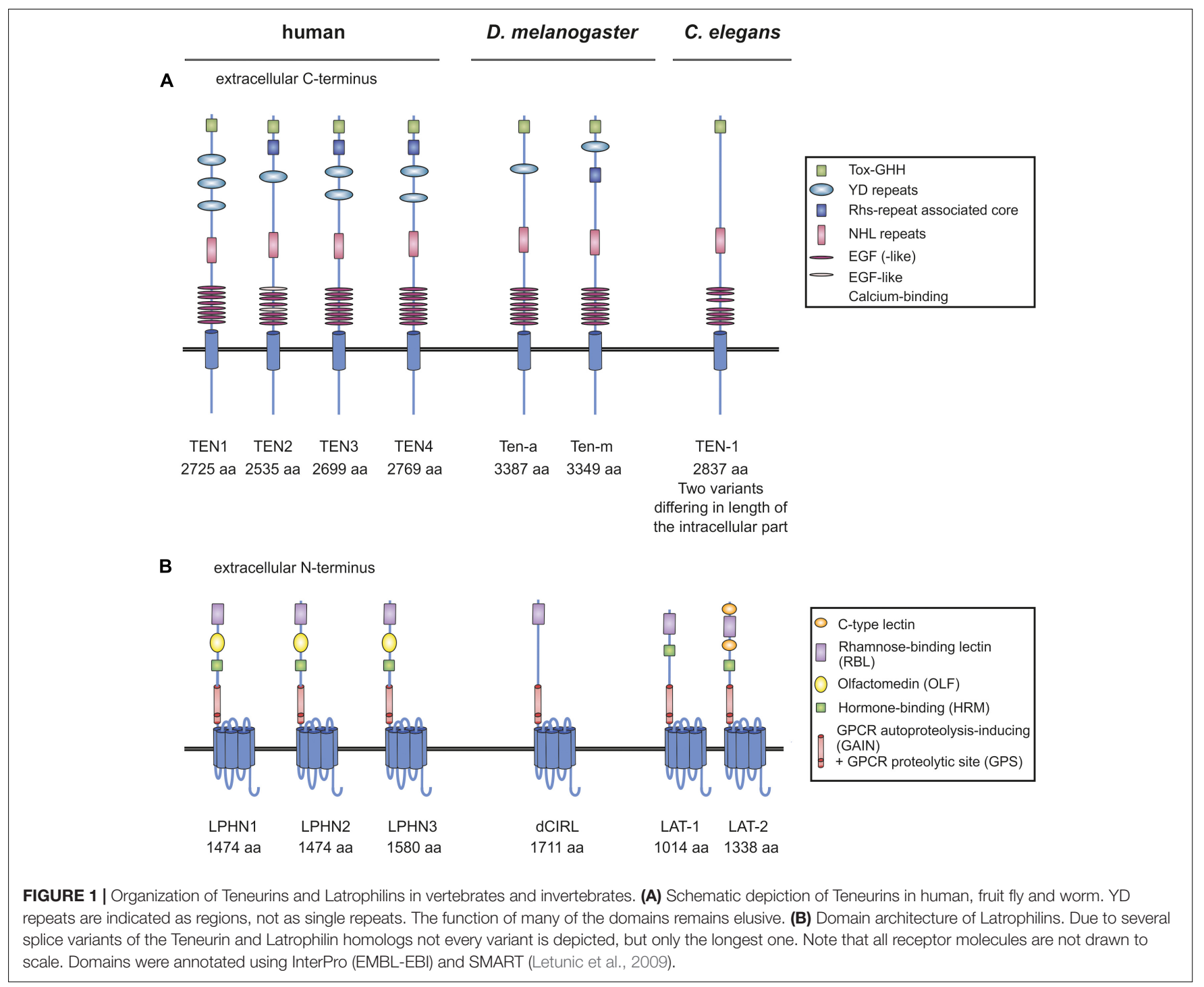

2018) indicating that LPHN1 is the receptor transducing information into the cell.

\section{LATROPHILINS AND TENEURINS IN INVERTEBRATES HAVE SIMILAR FUNCTIONS}

Due to the obvious relevance of the Latrophilin-Teneurin interaction in mammals the question of the generality of this partnership and thus, its conservation, arises. This question is especially of interest as invertebrate models are often used for elucidation of neurobiological aspects and understanding of association with pathologies. The described interaction between Latrophilins and Teneurins is so far limited to vertebrates, it has not been shown in invertebrate systems to date. However, Teneurins and Latrophilins are both highly conserved groups of molecules. First discovered in the fruit fly Drosophila melanogaster (Baumgartner and Chiquet-Ehrismann, 1993;
Levine et al., 1994), Teneurins are evolutionarily as old as the unicellular choanoflagellates and are present in all metazoa investigated so far (Tucker and Chiquet-Ehrismann, 2006; Tucker et al., 2012). Similarly, Latrophilins belong to the evolutionarily oldest groups of Adhesion GPCRs being present in vertebrates and in invertebrates. Functionally, Teneurins also seem to be highly conserved, not only in respect to their neuronal roles (sections "Latrophilins and Teneurins in D. melanogasterNo Evidence for Interaction" and "Latrophilins and Teneurins in C. elegans Development Do Not Function as Ligand-Receptor Pair"). The receptors also have functions beyond synapse formation. It has been shown in mice that TEN4 is required for mesoderm induction and gastrulation (Lossie et al., 2005; Nakamura et al., 2013). Consistently, non-neuronal expression of mammalian Teneurins is found during embryonic development. This pattern is similar to the one of the Caenorhabditis elegans ortholog, suggesting conserved non-neuronal functions (section "Latrophilins and Teneurins in C. elegans Development Do Not Function as Ligand-Receptor Pair"). In contrast, for all that 

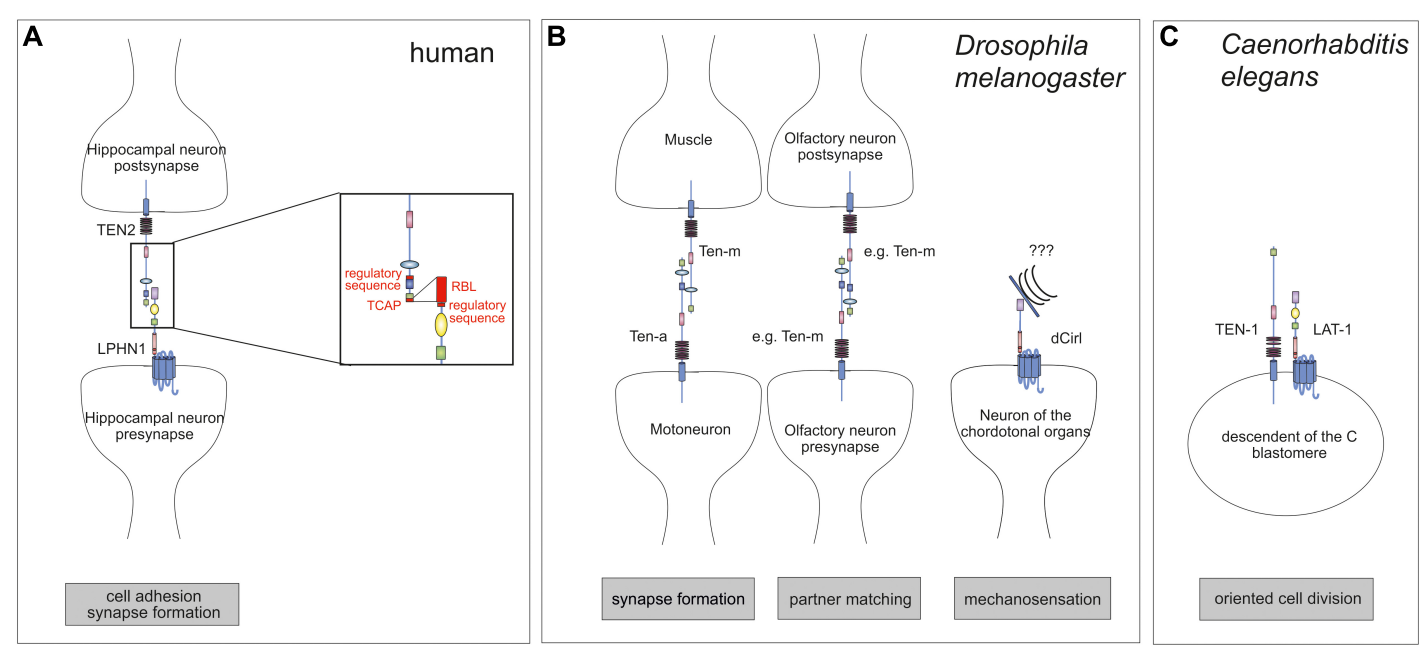

FIGURE 2 | Interaction of Teneurins and Latrophilins. (A) In mammals, the interaction between LPHN1 on the pre-synapse of hippocampal neurons with postsynaptic TEN2 contributes to the control of synapse formation. The interaction interfaces are roughly known. TEN2 binds to LPHN1 via the C-terminal portion of the Tox-GHH domain, the Teneurin C-terminal-associated peptide (TCAP). A short amino acid sequence further N-terminal is involved in regulation of the binding. On the LPHN1 side the rhamnose-binding lectin (RBL) is required for binding as well as a sequence between the RBL and olfactomedin (OLF) domains. (B) In the fruit fly, the Teneurins Ten-a and Ten-m interact heterophilically at the neuromuscular junction to ensure synapse formation. Further, a homophilic interaction between Teneurins controls partner matching for instance in the olfactory system. The only Latrophilin homolog in Drosophila, dCirl, is located on neurons of the chordotonal organs and are involved in mechanosensation. (C) The C. elegans homologs of Latrophilins, LAT-1, and Teneurin, TEN-1, are present on the same embryonic blastomeres, excluding the possibility of a classical ligand-receptor pair. Rather, they are acting in parallel. Note that it is rather likely that for any of the interactions shown additional molecules or dimerization are required which are not depicted here.

is known to date, the functional conservation of Latrophilins throughout phyla has not been shown beyond doubt (sections "Latrophilins and Teneurins in D. melanogaster-No Evidence for Interaction" and "Latrophilins and Teneurins in C. elegans Development Do Not Function as Ligand-Receptor Pair").

Due to the overall similar conservation of the two molecules it has been postulated that their interaction and its physiological impact are also evolutionarily old and conserved (Chand et al., 2013; Woelfle et al., 2015). Although experimental proof is lacking that in invertebrates Latrophilins and Teneurins interact, a body of functional proof in the invertebrate model organisms D. melanogaster and C. elegans exists suggesting that an interaction of the two is conceivable. However, there is also some information arguing against this assumption which will be discussed below.

\section{Latrophilins and Teneurins in D. melanogaster - No Evidence for Interaction}

Teneurins were first discovered in the fruit fly D. melanogaster as pair-rule genes tenascin-like molecule accessory (Ten-a) (Baumgartner and Chiquet-Ehrismann, 1993) and tenascin-like molecule major (Ten-m) (Baumgartner et al., 1994), which was also named odd oz (Odz) (Levine et al., 1994). The structural (Figure 1A) and functional conservation between mammalian and Drosophila Teneurins is evident. In Drosophila, Teneurins are widely expressed in neurons of the central and peripheral nervous system (Minet et al., 1999; Fascetti and Baumgartner, 2002) and several studies show their involvement in two different aspects at the synapse. Firstly, screens have revealed that they contribute to synapse formation of the neuromuscular junction (Liebl et al., 2006; Kurusu et al., 2008; Figure 2B). Further, Teneurins have implications in partner matching between presynaptic motoneurons and postsynaptic muscles as well as pre- and postsynaptic olfactory neurons and pre-synaptic motoneurons with postsynaptic muscles (Figure 2B; Hong et al., 2012; Mosca et al., 2012). Both functions can also be discriminated based on the connections that are formed by Teneurins. While in synaptogenesis Teneurins interact heterophilically (with another molecule or another Teneurin), they form homophilic interactions (with the same Teneurin) during partner matching. The heterophilic interaction partners described consist of presynaptic Ten-a and postsynaptic Ten-m and deletion of each of the molecules yields dysfunctional synapses and less synaptic boutons (Mosca et al., 2012). It is conceivable that Latrophilin might be another partner for a heterophilic interaction of Teneurins in this context. However, for Latrophilins, the functional conservation between mammals and Drosophila is not that evident, which is partly due the lack of knowledge about the receptor in the fruit fly. The one Latrophilin homolog the Drosophila genome carries, $d \mathrm{Cirl}$, has only been recently characterized. It is located on the neuronal dendrites and cilia of chordotonal organs in the fly and mediates sensitivity to touch (Scholz et al., 2015). This Adhesion GPCR is involved in mechanosenation, specifically shaping mechanically gated receptor currents by decreasing intracellular cyclic AMP levels, possibly by activating $G_{i}$ proteins (Scholz et al., 2017). The details of this function remain elusive and thus, no interaction with one of the Teneurins has been described so far. Such 
interaction can be debated because Ten-m/Odz and dCirl both are located in neurons of the chordotonal organs (Levine et al., 1997), but it seems that they are on the same cell rather than on opposing neurons. However, a partnership might still be likely as we are only just beginning to understand the functions of dCirl.

\section{Latrophilins and Teneurins in C. elegans Development Do Not Function as Ligand-Receptor Pair}

In the roundworm C. elegans, Teneurins and Latrophilins appear to have very similar functions and at first sight, it can be speculated that they form a classical interaction as described in mammals. However, a closer look prohibits this conclusion as of yet. In contrast to vertebrates or Drosophila, the nematode only has one Teneurin gene, ten-1. However, several transcript variants exist. The two most prominent ones are generated by two different transcription start sites: one variant with a longer (280 amino acids) intracellular domain and one with a short (36 amino acids) N-terminus (Drabikowski et al., 2005; Figure 1A). Both of these TEN-1 variants are present in distinct subsets of neurons (Drabikowski et al., 2005). Consistently, a role for TEN-1 in neuronal pathfinding has been postulated (Drabikowski et al., 2005). Although lat-1, one of the two Latrophilin homologs in C. elegans (Figure 1B), is also expressed in neurons (Langenhan et al., 2009), so far no neuronal function of LAT-1 has been described leading to the question whether the classical Latrophilin-Teneurin interaction plays a role in C. elegans. LAT-1 is functionally highly diverse. It has roles in fertility and cell polarity during development (Langenhan et al., 2009; Prömel et al., 2012), where it elicits a $\mathrm{G}_{\mathrm{s}}$ protein-mediated signal raising intracellular cyclic AMP levels (Müller et al., 2015), but a role in synaptogenesis similar to the one in mammals, has not been described yet, precluding a final assessment.

However, similar to mammalian and Drosophila Teneurins, expression of ten-1 is not limited to neuronal tissues but is also found in hypodermal cells (long TEN-1 variant), in cells of the gut, the somatic gonad, distal tip cell, and in few muscle cells (short TEN-1 variant) (Drabikowski et al., 2005). Interestingly, the expression pattern in non-neuronal cells is almost identical to the one of lat-1, which is mainly confined to cells of the somatic gonad and the distal tip cell (Langenhan et al., 2009), suggesting that LAT-1 and TEN-1 might have similar functions in a non-neuronal context. Indeed, not only the expression pattern of lat-1 and ten-1 is highly similar, but also the phenotype that respective knockout mutants display. Both mutants lat-1(ok1465) and ten-1(ok641) exhibit morphogenesis defects (Drabikowski et al., 2005; Langenhan et al., 2009). However, genetic analyses revealed that both genes act in parallel during development implying a synergistic rather than linear interaction between lat-1 and ten-1 (Langenhan et al., 2009; Figure 2C). In line with these findings, expression data show localization of TEN-1 and LAT1 on the same embryonic blastomeres rather than on opposing cells, indicating that the two receptors do not form the classical ligand-receptor pair on two different cells in C. elegans (Prömel et al., 2012). However, since it is conceivable that Teneurins have multiple functions beyond their role in neurons, it cannot be fully excluded that for some other function, a classical interaction with Latrophilins is required. Further, the second Latrophilin homolog in C. elegans, lat-2, has not been functionally characterized yet and thus, might also be a candidate for a partnership with TEN-1.

\section{AN EVOLUTIONARY VIEW ON LATROPHILINS AND TENEURINS POINTS TOWARD A YOUNG INTERACTION}

Due to their similar expression and function in vertebrates and invertebrates and their high conservation it has been speculated that the Latrophilin-Teneurin interaction also exists in invertebrates (Woelfle et al., 2015). Indeed, a high general sequence conservation of Teneurins from choanoflagellates to vertebrates has been found (Tucker and Chiquet-Ehrismann, 2006) together with structural conservation of core folds and several domains (Jackson et al., 2018; Li et al., 2018). Parts of the Teneurin N-terminus are probably derived from an evolutionarily ancient YD-repeat shell domain that is widespread across the bacterial kingdom by horizontal gene transfer into an early metazoan genome (Jackson et al., 2018). The EGF domains of the Teneurin N-terminus appear first in multicellular animals. Further, comparison of the gene organization among human Ten1, Drosophila Ten- $a$ and Ten- $m$ and the C. elegans ten-1 revealed the presence of both, conserved intron locations and exon sequences (Minet and Chiquet-Ehrismann, 2000; Tucker et al., 2012), suggesting that Teneurins arose from a single ancestral gene. This high structural and sequence conservation points toward comparable functions of Teneurins in similar molecular contexts in different species. However, a closer look at the evolution of Latrophilins can cast doubt on the hypothesis that the interaction of Teneurins with Latrophilins is old.

\section{Invertebrate Latrophilins Are Not One-to-One Orthologs of Mammalian Latrophilins}

The class of Adhesion GPCRs belongs to the oldest GPCRs and their sequence signatures in the 7TM part appear first in unicellular organisms such as Dictyostelium discoideum and fungi (Krishnan et al., 2012). It needs to be noted that the appearance of genes in unicellular organisms should be taken with caution in the analysis of evolutionary history of gene families due to the possibility of horizontal gene transfer. However, in evolutionarily basal animals such as placozoa (Trichoplax adhaerens) and choanoflagellates (Salpingoeca rosetta and Monosiga brevicollis) there is already a number of Adhesion GPCR-encoding genes indicating their stable integration into animal genomes. Due to high sequence distances it is hard to assign them to Latrophilins or to another of the eight distinct groups of vertebrate Adhesion GPCRs (Nordstrom et al., 2011). Furthermore, none of these evolutionarily old Adhesion GPCRs have been found to present themselves with an RBL-, an OLF-, or an HRM domain (Krishnan et al., 2012), which have been suggested to interact with Teneurins (Woelfle et al., 2015). Therefore, it is rather unlikely that 

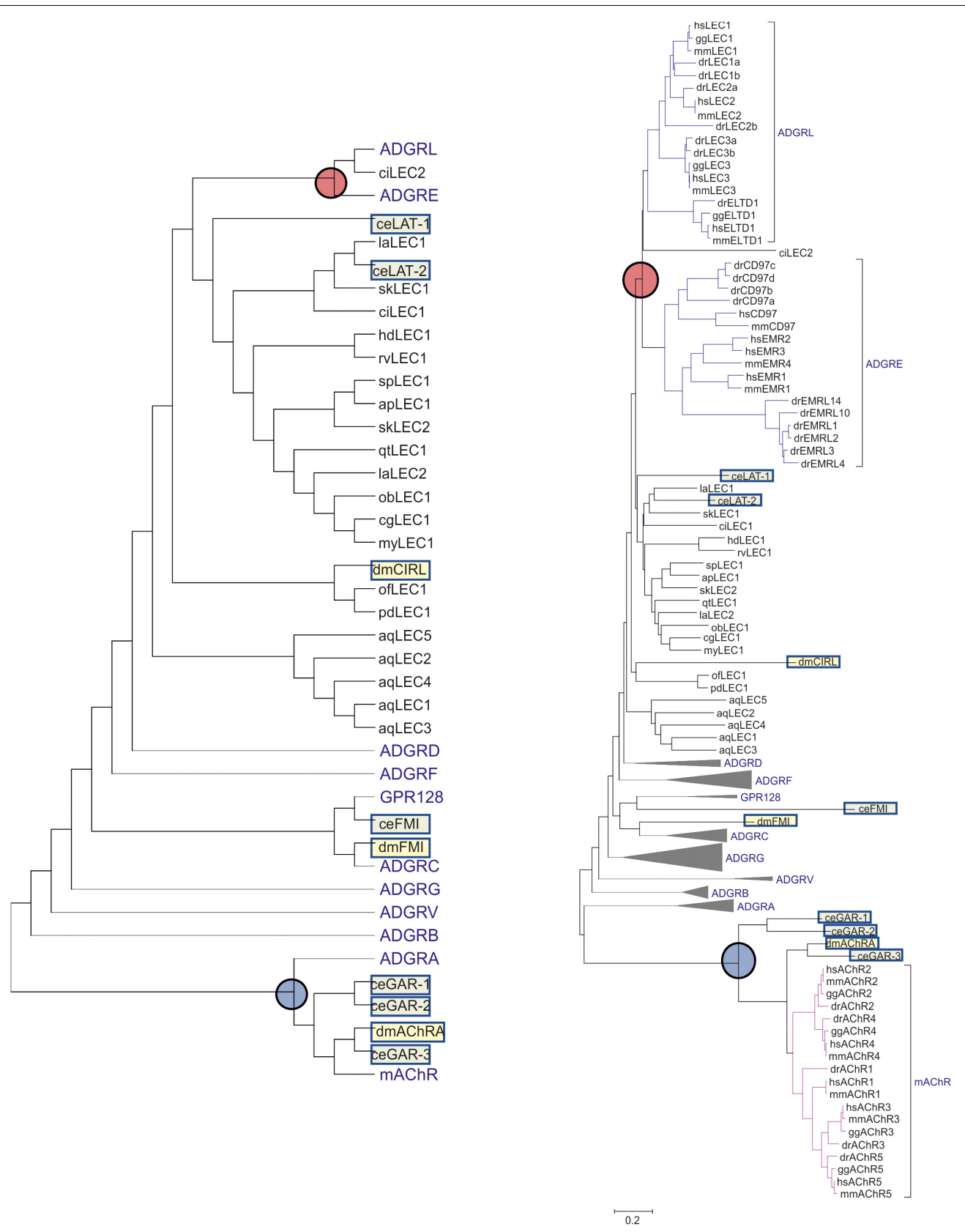

FIGURE 3 | Evolutionary relationships of Latrophilins with other Adhesion GPCR groups. The evolutionary history of C. elegans (ce, light green box) and Drosophila melanogaster (dm, light yellow box) Latrophilin-like (LAT, Cirl, LEC) and Flamingo-like (FMI) receptors in relation to human, mouse, chicken, and zebrafish Adhesion GPCRs was constructed using the amino acid sequence of the 7TM region. For comparison purposes, Latrophilin-like sequences from other invertebrates were included. Muscarinic acetyl choline receptors of the respective species were used as outgroup. All sequence data are retrieved from NCBI. The evolutionary history was inferred using the Neighbor-Joining method (Saitou and Nei, 1987). The optimal tree with the sum of branch length $=38.4$ is shown. The evolutionary distances were computed using the Poisson correction method (Zuckerkandl and Pauling, 1965) and are shown as units of the number of amino acid substitutions per site. The analysis involved 180 amino acid sequences. All positions with less than $95 \%$ site coverage were eliminated. That is, fewer than $5 \%$ alignment gaps, missing data, and ambiguous bases were allowed at any position. There were a total of 202 positions in the final dataset. Evolutionary analyses were conducted in MEGA7 (Kumar et al., 2016). The left, less complex tree shows only the topology of the groups. The respective right tree displays the calculated branch lengths but the receptor groups are condensed into proportional triangles except of ADGRL, ADGRE and the muscarinic acetylcholine receptors (mAChR). The branch point of ADGRL and ADGRE is marked with a red circle. The mAChR branch is marked with a blue circle. Species are: hs, Homo sapiens (Mammalia); mm, Mus musculus (Mammalia); gg, Gallus gallus (Avea); dr, Danio rerio (Osteichthyes); sk, Saccoglossus kowalevskii (Hemichordata); ci, Ciona intestinalis (Tunicata); ac, Acanthaster planc (Echinodermata); sp, Strongylocentrotus purpuratus (Echinodermata); my, Mizuhopecten yessoensis (Mollusca); cg, Crassostrea gigas (Mollusca); ob, Octopus bimaculoides (Mollusca); dm, Drosophila melanogaster (Insecta); la, Lingula anatina (Brachiopoda); ct, Capitella teleta (Annelida); ce, Caenorhabditis elegans (Nematoda); hd, Hypsibius dujardini (Tardigrada); rv, Ramazzottius varieornatus (Tardigrada); of, Orbicella faveolata (Cnidaria); pd, Pocillopora damicornis (Cnidaria); aq, Amphimedon queenslandica (Parazoa). 
a functional paring of Adhesion GPCRs and Teneurin-like proteins, as described in vertebrates, was already established at this early evolutionary stage, although Teneurin-like proteins are present in placozoa and choanoflagellates.

In the genomes of the roundworm $C$. elegans and the fruit fly D. melanogaster, two and one Latrophilin genes, respectively, have been assigned based on sequence similarities in the $\mathrm{N}$-terminus and the 7TM domain. Re-evaluation of the already described phylogenetic relationship of these invertebrate and vertebrate Adhesion GPCRs (Schioth et al., 2010) revealed a more complex picture placing the 7TM domains of the C. elegans Latrophilins LAT-1 and LAT-2 basal to both, the vertebrate
Latrophilin (ADGRL) and EMR (ADGRE) groups (Figure 3). In tunicates and evolutionarily old chordates such as lancelet (Branchiostoma belcheri) there are obviously no orthologs or paralogs of the ADGRE group, which contains EMR14 (ADGRE1-4) and CD97 (ADGRE5) (Figure 3). However, as these can be found in fishes, one can assume that the ADGRE group evolved from the ADGRL group [containing besides LPHN1-3 also ELTD1 (ADGRL4)] in early vertebrate evolution or, alternatively, but more unlikely, was eliminated from all invertebrates. Therefore, the 7TM of LAT-1 and LAT2 from C. elegans and other invertebrates are not in oneto-one orthology to vertebrate Latrophilins but rather share

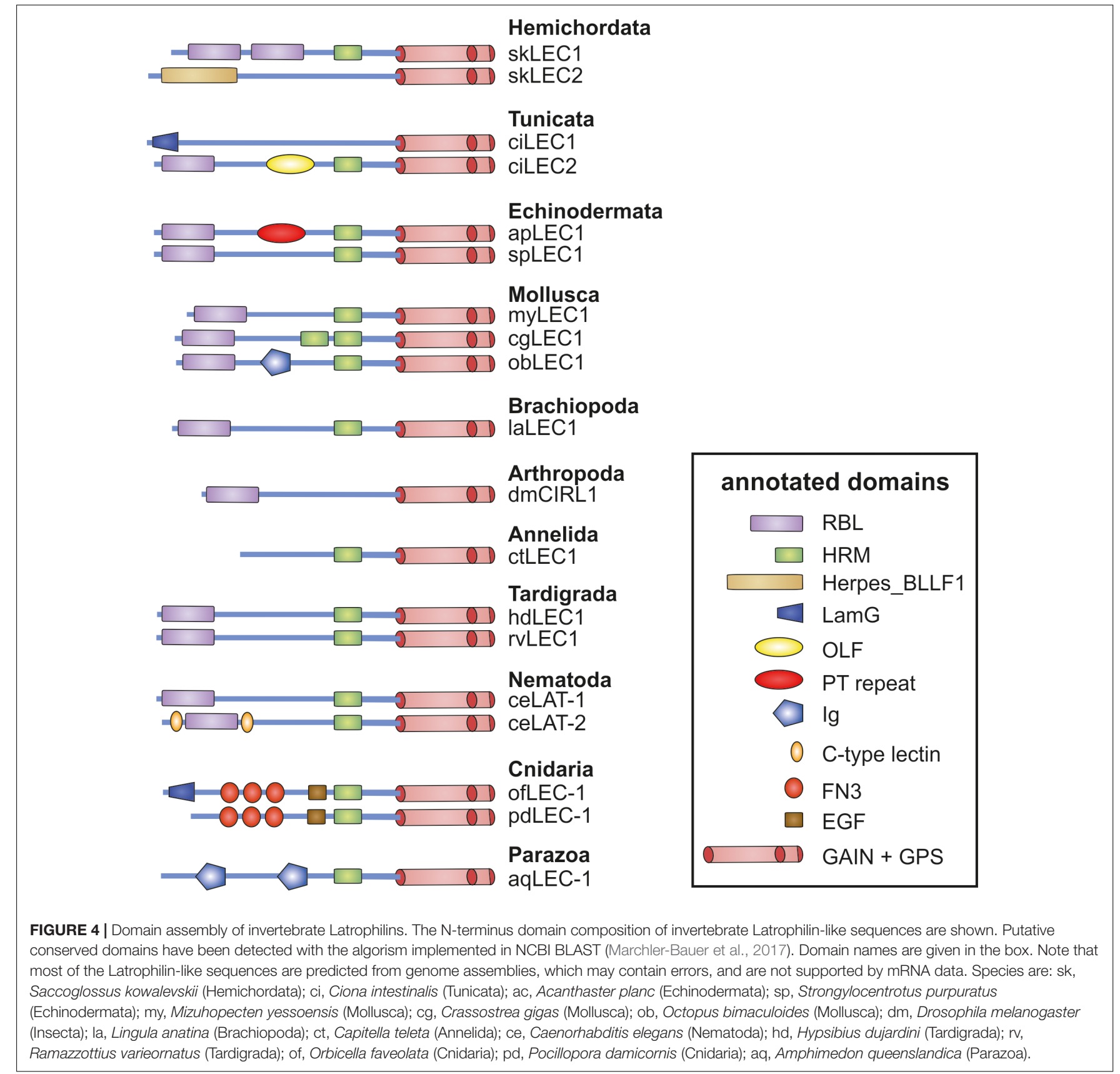


phylogenetic relation to all members of both groups including Latrophilins, ELTD, EMRs, and CD97. Further, based on substitution rates, C. elegans lat-1 and lat-2 and most invertebrate Latrophilin-like sequences are even more distantly related to the vertebrate Adhesion GPCR groups ADGRL and ADGRE than the $C$. elegans muscarinic acetylcholine receptors gar-1/-2/-3 to their vertebrate orthologs/paralogs (Figure 3). Most interestingly, the fruit fly Latrophilin dCirl is even more distantly related to the ADGRL group being placed closer to the Latrophilin-like sequences of Cnidaria and Parazoa and other Adhesion GPCR groups (Figure 3). Phylogenetic relation built on the basis of the 7TM sequences provides only weak support considering dCirl a member of the Latrophilin group at all. Even if the extracellular N-terminus and its modular composition presents with some structural features of the Latrophilin group, the very distant relation of the 7TM domain may explain differences in their $G$ protein-mediated signal transduction in different species (Lelianova et al., 1997; Müller et al., 2015; Scholz et al., 2017; Nazarko et al., 2018). It has to be noted that already the five vertebrate muscarinic acetylcholine receptors (represented in the lilac triangle in Figure 3) differ in their signaling properties by coupling to $\mathrm{G}_{\mathrm{q} / 11}(\mathrm{mAChR}-1,-3,-5)$ and $\mathrm{G}_{\mathrm{i} / \mathrm{o}}(\mathrm{mAChR}-2,-4)$.

\section{The Postulated Teneurin-Latrophilin Interaction Sites Are Not Evolutionarily Old}

Although the phylogenetic analyses on Latrophilins argue at least against the receptor binding to Teneurin and eliciting a conserved signal into the cells, it is still conceivable that an interaction between invertebrate Latrophilins and Teneurins occurs with Latrophilins acting as ligands for Teneurins. The interaction of Latrophilins and Teneurins is mediated by their $\mathrm{N}$-termini and, taking this thought further, one can hypothesize that the 7TM is only modularly attached mediating the appropriated intracellular signal in the different species. As already seen in Figure 1, the worm LAT-1/LAT-2 and the fruit fly dCirl N-termini do not contain an OLF domain and additionally, the HRM domain is missing in dCirl. Detailed analysis of the Latrophilin N-termini in currently available genomes revealed that the ensemble of RBL-, OLF-, and HRM domains in the N-termini of Adhesion GPCRs is found in tunicates (e.g., Ciona intestinalis) (Figure 4), in lancelet (Branchiostoma belcheri), and Chondrichthyes (Callorhinchus milii). In Hemichordata, Echinodermata, Mollusca, Nematoda, Arthropoda, Tardigrada, and Brachiopoda only the GAIN, RBL, and HRM domain (sometimes degenerated or absent) are mostly present (Figure 4), but none of these sequences contains an OLF domain. Interestingly, several invertebrate Latrophilins contain domains (e.g., EGF, Ig, LamG, and FN3) not seen in vertebrate Latrophilins (Figure 4), indicating a modular structure of these Adhesion GPCRs.

Analyses on Latrophilin-Teneurin interactions provide strong evidence that the main site of interaction is the RBL domain with contribution of a short sequence C-terminal of the domain (Figure 2A; Silva et al., 2011; Boucard et al., 2014). Although protein domain identification tools constantly assign RBL and HRM domains in Latrophilins, the amino acid sequence conservation is low (Figure 5). The

\section{A}
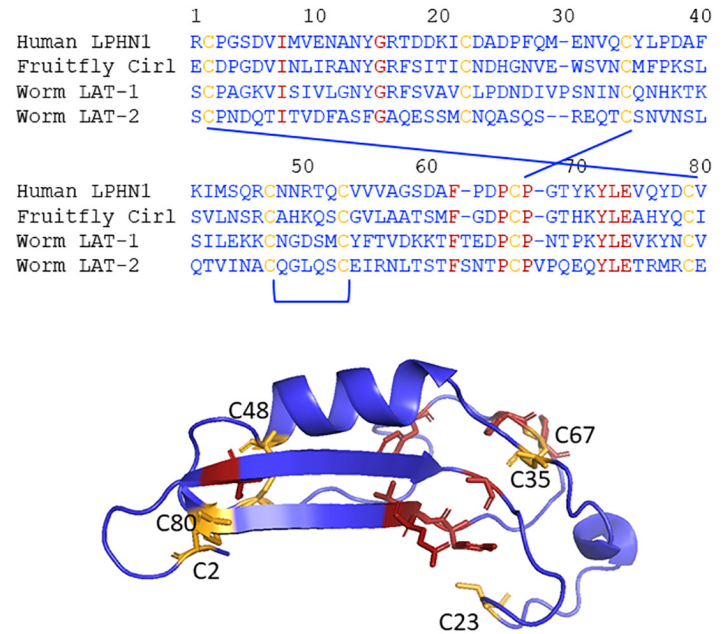

B

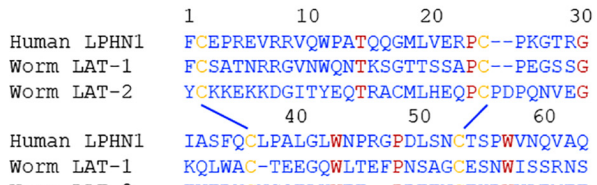
$\begin{array}{ll}\text { Worm LAT-1 } & \text { KQLWAC-TEEGQWLTEFPNSAGCESNWISSRNS } \\ \text { Worm LAT-2 } & \text { TVTRYCNCQTAKWET--PDTTNCTHRWVAEMET }\end{array}$

RBL

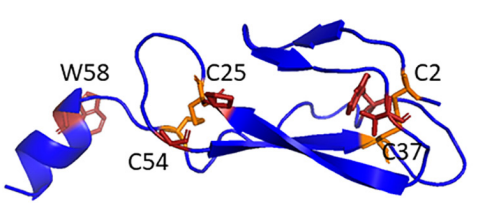

HRM

FIGURE 5 | Low evolutionary conservation of the RBL and HMR domains. The amino acid sequence alignments of the putative RBL (A) and the HRM (B) domains of human LPHN1, fruit fly dCirl and C. elegans LAT-1 and LAT-2 are shown. The conserved cysteine (yellow) and other (red) residues are highlighted. Homology models of the three-dimensional structures of the human LPHN1 RBL and HRM domains were generated using Phyre2 (Kelley et al., 2015 ) based on the best matching templates pdb: c5afbA and pdb: c4dlqA, respectively. Again, the conserved cysteine (yellow) and other (red) residues are highlighted. 
domain assignment is mainly based on conserved disulfide bond-forming cysteine residues keeping constant folds of the domains. The few other conserved residues mainly surround the conserved disulfide bonds (Figure 5). This suggests that these backbone structures provide the threedimensional scaffold of the RBL and HRM domains. The remaining amino acid residues most probably participate in specific functions of the two domains. One can speculate that these domains mediate low affinity interactions to proteins or compound or that the sequence variability is the result of a co-evolutionary process with an also variable interaction partner. Although it cannot be fully excluded that invertebrate Latrophilins interact with Teneurins, it does not appear to be likely based on the re-evaluation of existing data above.

\section{CONCLUSION AND FUTURE PERSPECTIVES}

Synapse formation is a highly complex and tightly regulated process and although several aspects have been already well understood, many details are still obscure. Latrophilins and Teneurins are both transmembrane proteins which have been described to have implications in synaptogenesis and synapse function. While for Teneurins this has been shown in vertebrate and invertebrate systems, a lot of information is still lacking for Latrophilins. However, a transsynaptic interaction of the two is essential for adhesion and synapse formation in mammals. The question of whether this interaction represents a common principle in the generation of synapses throughout phyla remains unanswered, mainly due to lacking experimental evidence, but is highly intriguing. Similarities in expression, seemingly functional redundancy in Drosophila and a general evolutionary conservation makes it tempting to conclude that this transsynaptic interaction is old and also meaningful in invertebrate species. However, a closer look at phylogenetic evidence and existing data sheds light on a different picture.

Our phylogenetic analyses indicate that, although basal metazoans already contain Adhesion GPCR, clearly Latrophilin7TM-related sequences only appeared at the level of Eumetazoa and thus, later in evolution than Teneurins. The connected $\mathrm{N}$-termini contain RBL- and HRM-like domains but not constantly. Further, the OLF domain only appears in the $\mathrm{N}$-termini of Latrophilins in early chordate evolution. Although the conserved cysteine bonds and a few other conserved positions allow for assignments as RBL and HRM domains, most of the remaining sequence is highly variable in these domains. This indicates that the RBL and HRM domains in Latrophilins may have specific functions in the different species and/or underwent co-evolution with interaction partners rather than mediating evolutionarily conserved protein-protein interactions. This analysis yields some evidence that a conserved interaction of Latrophilins and Teneurins in invertebrates might not be likely. It cannot be excluded that additional, not yet identified interaction sites in Latrophilins exist, which represent highly conserved sequences. Further, the role of other proteins or molecules aiding or promoting the interaction cannot be evaluated. For instance, dystroglycans have been discussed to be part of a larger complex (Woelfle et al., 2015). However, if a physical interaction may occur, a potential signal elicited by the Adhesion GPCR is not comparable to signals transduced by mammalian Latrophilins as invertebrate Latrophilins, in particular the homolog in Drosophila, are not one-to-one homologs of mammalian Latrophilins, but also bear resemblance to other Adhesion GPCRs. This argument is further supported by experimental data highlighting distinct signaling cascades activated by Latrophilin homologs of different species: While mammalian LPHN1 can signal via $G_{s}$ or $G_{i}$ proteins (Müller et al., 2015; Nazarko et al., 2018), Drosophila dCirl activates $G_{i}$ proteins and C. elegans LAT-1 $\mathrm{G}_{\mathrm{s}}$ proteins. A functional evaluation of these different cascades will shed light on the impact of these cascades.

We cannot exclude an interaction between Latrophilins and Teneurins in invertebrates, however, the mode of interaction might be realized differently from their mammalian counterparts. While both groups of proteins have essential functions in invertebrates and the ones of Teneurins in particular are highly conserved roles across phyla, they might not realize this role via the help of Latrophilins. Invertebrates have less complex regulatory circuits and hence, different requirements for synapse formation and function. Thus, it would not be surprising that they utilize different mechanisms to establish and maintain synapses and their function.

Future analyses need to focus on gaining a better understanding of the physiological functions mediated by both, Latrophilins and Teneurins, in mammals and invertebrates. These will help understand similarities as well as differences in the function of each receptor in different contexts and aid the understanding of the molecular mechanisms underlying synaptogenesis and neuronal wiring in vertebrates and invertebrates. It will be highly interesting to gain information on the existence and composition of potential synaptic complexes involving Latrophilins and/or Teneurins. Further, identifying interaction interfaces of mammalian Latrophilins with Teneurins can be highly informative for the prediction and characterization of a potential interaction in other species.

\section{AUTHOR CONTRIBUTIONS}

TS and SP researched and wrote the manuscript.

\section{FUNDING}

This work was supported by the European Social Fund and the German Research Foundation (DFG: FOR 2149/P02 and P04, SFB 1052/B6). 


\section{REFERENCES}

Al Chawaf, A., St Amant, K., Belsham, D., and Lovejoy, D. A. (2007a). Regulation of neurite growth in immortalized mouse hypothalamic neurons and rat hippocampal primary cultures by teneurin C-terminal-associated peptide-1. Neuroscience 144, 1241-1254. doi: 10.1016/j.neuroscience.2006.09.062

Al Chawaf, A., Xu, K., Tan, L., Vaccarino, F. J., Lovejoy, D. A., and Rotzinger, S. (2007b). Corticotropin-releasing factor (CRF)-induced behaviors are modulated by intravenous administration of teneurin C-terminal associated peptide-1 (TCAP-1). Peptides 28, 1406-1415. doi: 10.1016/j.peptides.2007. 05.014

Anderson, G. R., Maxeiner, S., Sando, R., Tsetsenis, T., Malenka, R. C., and Sudhof, T. C. (2017). Postsynaptic adhesion GPCR latrophilin-2 mediates target recognition in entorhinal-hippocampal synapse assembly. J. Cell Biol. 216, 3831-3846. doi: $10.1083 /$ jcb.201703042

Antinucci, P., Nikolaou, N., Meyer, M. P., and Hindges, R. (2013). Teneurin-3 specifies morphological and functional connectivity of retinal ganglion cells in the vertebrate visual system. Cell Rep. 5, 582-592. doi: 10.1016/j.celrep.2013. 09.045

Arcos-Burgos, M., Jain, M., Acosta, M. T., Shively, S., Stanescu, H., Wallis, D., et al. (2010). A common variant of the latrophilin 3 gene, LPHN3, confers susceptibility to ADHD and predicts effectiveness of stimulant medication. Mol. Psychiatry 15, 1053-1066. doi: 10.1038/mp.2010.6

Baumgartner, S., and Chiquet-Ehrismann, R. (1993). Tena, a Drosophila gene related to tenascin, shows selective transcript localization. Mech. Dev. 40, 165-176. doi: 10.1016/0925-4773(93)90074-8

Baumgartner, S., Martin, D., Hagios, C., and Chiquet-Ehrismann, R. (1994). Tenm, a Drosophila gene related to tenascin, is a new pair-rule gene. EMBO J. 13, 3728-3740. doi: 10.1002/j.1460-2075.1994.tb06682.x

Berns, D. S., DeNardo, L. A., Pederick, D. T., and Luo, L. (2018). Teneurin3 controls topographic circuit assembly in the hippocampus. Nature 554, 328-333. doi: 10.1038/nature25463

Boucard, A. A., Ko, J., and Sudhof, T. C. (2012). High affinity neurexin binding to cell adhesion G-protein-coupled receptor CIRL1/latrophilin-1 produces an intercellular adhesion complex. J. Biol. Chem. 287, 9399-9413. doi: 10.1074/jbc. M111.318659

Boucard, A. A., Maxeiner, S., and Sudhof, T. C. (2014). Latrophilins function as heterophilic cell-adhesion molecules by binding to teneurins: regulation by alternative splicing. J. Biol. Chem. 289, 387-402. doi: 10.1074/jbc.M113.504779

Chand, D., de Lannoy, L., Tucker, R., and Lovejoy, D. A. (2013). Origin of chordate peptides by horizontal protozoan gene transfer in early metazoans and protists: evolution of the teneurin C-terminal associated peptides (TCAP). Gen. Comp. Endocrinol. 188, 144-150. doi: 10.1016/j.ygcen.2013.02.006

Chand, D., Song, L., deLannoy, L., Barsyte-Lovejoy, D., Ackloo, S., Boutros, P. C., et al. (2012). C-Terminal region of teneurin-1 co-localizes with dystroglycan and modulates cytoskeletal organization through an extracellular signalregulated kinase-dependent stathmin- and filamin A-mediated mechanism in hippocampal cells. Neuroscience 219, 255-270. doi: 10.1016/j.neuroscience. 2012.05.069

Drabikowski, K., Trzebiatowska, A., and Chiquet-Ehrismann, R. (2005). ten-1, an essential gene for germ cell development, epidermal morphogenesis, gonad migration, and neuronal pathfinding in Caenorhabditis elegans. Dev. Biol. 282, 27-38. doi: 10.1016/j.ydbio.2005.02.017

Erb, S., McPhee, M., Brown, Z. J., Kupferschmidt, D. A., Song, L., and Lovejoy, D. A. (2014). Repeated intravenous administrations of teneurin-C terminal associated peptide (TCAP)-1 attenuates reinstatement of cocaine seeking by corticotropin-releasing factor (CRF) in rats. Behav. Brain Res. 269, 1-5. doi: 10.1016/j.bbr.2014.04.013

Fascetti, N., and Baumgartner, S. (2002). Expression of Drosophila Ten-a, a dimeric receptor during embryonic development. Mech. Dev. 114, 197-200. doi: 10. 1016/S0925-4773(02)00055-2

Hong, W., Mosca, T. J., and Luo, L. (2012). Teneurins instruct synaptic partner matching in an olfactory map. Nature 484, 201-207. doi: 10.1038/nature10926

Jackson, V. A., del Toro, D., Carrasquero, M., Roversi, P., Harlos, K., Klein, R., et al. (2015). Structural basis of latrophilin-FLRT interaction. Structure 23, 774-781. doi: 10.1016/j.str.2015.01.013

Jackson, V. A., Meijer, D. H., Carrasquero, M., van Bezouwen, L. S., Lowe, E. D., Kleanthous, C., et al. (2018). Structures of Teneurin adhesion receptors reveal an ancient fold for cell-cell interaction. Nat. Commun. 9:1079. doi: 10.1038/ s41467-018-03460-0

Kelley, L. A., Mezulis, S., Yates, C. M., Wass, M. N., and Sternberg, M. J. (2015). The Phyre2 web portal for protein modeling, prediction and analysis. Nat. Protoc. 10, 845-858. doi: 10.1038/nprot.2015.053

Kenzelmann, D., Chiquet-Ehrismann, R., Leachman, N. T., and Tucker, R. P. (2008). Teneurin-1 is expressed in interconnected regions of the developing brain and is processed in vivo. BMC Dev. Biol. 8:30. doi: 10.1186/1471213X-8-30

Krasnoperov, V. G., Beavis, R., Chepurny, O. G., Little, A. R., Plotnikov, A. N., and Petrenko, A. G. (1996). The calcium-independent receptor of alpha-latrotoxin is not a neurexin. Biochem. Biophys. Res. Commun. 227, 868-875. doi: 10.1006/ bbrc. 1996.1598

Krishnan, A., Almen, M. S., Fredriksson, R., and Schioth, H. B. (2012). The origin of GPCRs: identification of mammalian like Rhodopsin, Adhesion, Glutamate and Frizzled GPCRs in fungi. PLoS One 7:e29817. doi: 10.1371/journal.pone. 0029817

Kumar, S., Stecher, G., and Tamura, K. (2016). MEGA7: molecular evolutionary genetics analysis version 7.0 for bigger datasets. Mol. Biol. Evol. 33, 1870-1874. doi: $10.1093 / \mathrm{molbev} / \mathrm{msw} 054$

Kurusu, M., Cording, A., Taniguchi, M., Menon, K., Suzuki, E., and Zinn, K. (2008). A screen of cell-surface molecules identifies leucine-rich repeat proteins as key mediators of synaptic target selection. Neuron 59, 972-985. doi: 10.1016/ j.neuron.2008.07.037

Lange, M., Norton, W., Coolen, M., Chaminade, M., Merker, S., Proft, F., et al. (2012). The ADHD-susceptibility gene lphn3.1 modulates dopaminergic neuron formation and locomotor activity during zebrafish development. Mol. Psychiatry 17, 946-954. doi: 10.1038/mp.2012.29

Langenhan, T., Prömel, S., Mestek, L., Esmaeili, B., Waller-Evans, H., Hennig, C., et al. (2009). Latrophilin signaling links anterior-posterior tissue polarity and oriented cell divisions in the C. elegans embryo. Dev. Cell 17, 494-504. doi: 10.1016/j.devcel.2009.08.008

Leamey, C. A., Merlin, S., Lattouf, P., Sawatari, A., Zhou, X., Demel, N., et al. (2007). Ten_m3 regulates eye-specific patterning in the mammalian visual pathway and is required for binocular vision. PLoS Biol. 5:e241. doi: 10.1371/ journal.pbio.0050241

Lelianova, V. G., Davletov, B. A., Sterling, A., Rahman, M. A., Grishin, E. V., Totty, N. F., et al. (1997). Alpha-latrotoxin receptor, latrophilin, is a novel member of the secretin family of G protein-coupled receptors. J. Biol. Chem. 272, 21504-21508. doi: 10.1074/jbc.272.34.21504

Letunic, I., Doerks, T., and Bork, P. (2009). SMART 6: recent updates and new developments. Nucleic Acids Res. 37, D229-D232. doi: 10.1093/nar/gkn808

Levine, A., Bashan-Ahrend, A., Budai-Hadrian, O., Gartenberg, D., Menasherow, S., and Wides, R. (1994). Odd Oz: a novel Drosophila pair rule gene. Cell 77, 587-598. doi: 10.1016/0092-8674(94)90220-8

Levine, A., Weiss, C., and Wides, R. (1997). Expression of the pair-rule gene odd Oz (odz) in imaginal tissues. Dev. Dyn. 209, 1-14. doi: 10.1002/(SICI)10970177(199705)209:1<1::AID-AJA1>3.0.CO;2-M

Li, J., Shalev-Benami, M., Sando, R., Jiang, X., Kibrom, A., Wang, J., et al. (2018). Structural basis for Teneurin function in circuit-wiring: a toxin motif at the synapse. Cell 173, 735.e15-748.e15. doi: 10.1016/j.cell.2018.03.036

Liebl, F. L., Werner, K. M., Sheng, Q., Karr, J. E., McCabe, B. D., and Featherstone, D. E. (2006). Genome-wide P-element screen for Drosophila synaptogenesis mutants. J. Neurobiol. 66, 332-347. doi: 10.1002/neu.20229

Lossie, A. C., Nakamura, H., Thomas, S. E., and Justice, M. J. (2005). Mutation of $17 \mathrm{Rn} 3$ shows that Odz4 is required for mouse gastrulation. Genetics 169, 285-299. doi: 10.1534/genetics.104.034967

Lu, Y. C., Nazarko, O. V., Sando, R. III, Salzman, G. S., Li, N. S., Sudhof, T. C., et al. (2015). Structural basis of latrophilin-FLRT-UNC5 interaction in cell adhesion. Structure 23, 1678-1691. doi: 10.1016/j.str.2015.06.024

Marchler-Bauer, A., Bo, Y., Han, L., He, J., Lanczycki, C. J., Lu, S., et al. (2017). CDD/SPARCLE: functional classification of proteins via subfamily domain architectures. Nucleic Acids Res. 45, D200-D203. doi: 10.1093/nar/gkw1129

Minet, A. D., and Chiquet-Ehrismann, R. (2000). Phylogenetic analysis of teneurin genes and comparison to the rearrangement hot spot elements of E. coli. Gene 257, 87-97. doi: 10.1016/S0378-1119(00)00388-7

Minet, A. D., Rubin, B. P., Tucker, R. P., Baumgartner, S., and ChiquetEhrismann, R. (1999). Teneurin-1, a vertebrate homologue of the Drosophila 
pair-rule gene ten-m, is a neuronal protein with a novel type of heparin-binding domain. J. Cell Sci. 112(Pt 12), 2019-2032.

Mosca, T. J. (2015). On the Teneurin track: a new synaptic organization molecule emerges. Front. Cell. Neurosci. 9:204. doi: 10.3389/fncel.2015.00204

Mosca, T. J., Hong, W., Dani, V. S., Favaloro, V., and Luo, L. (2012). Trans-synaptic Teneurin signalling in neuromuscular synapse organization and target choice. Nature 484, 237-241. doi: 10.1038/nature10923

Müller, A., Winkler, J., Fiedler, F., Sastradihardja, T., Binder, C., Schnabel, R., et al. (2015). Oriented cell division in the C. elegans embryo is coordinated by G-Protein signaling dependent on the adhesion GPCR LAT-1. PLoS Genet. 11:e1005624. doi: 10.1371/journal.pgen.1005624

Nakamura, H., Cook, R. N., and Justice, M. J. (2013). Mouse Tenm4 is required for mesoderm induction. BMC Dev. Biol. 13:9. doi: 10.1186/1471-213X-13-9

Nazarko, O., Kibrom, A., Winkler, J., Leon, K., Stoveken, H., Salzman, G., et al. (2018). A comprehensive mutagenesis screen of the adhesion GPCR Latrophilin-1/ADGRL1. iScience 3, 264-278. doi: 10.1016/j.isci.2018.04.019

Nordstrom, K. J., Sallman Almen, M., Edstam, M. M., Fredriksson, R., and Schioth, H. B. (2011). Independent HHsearch, Needleman-Wunsch-based, and motif analyses reveal the overall hierarchy for most of the $\mathrm{G}$ protein-coupled receptor families. Mol. Biol. Evol. 28, 2471-2480. doi: 10.1093/molbev/msr061

Nozumi, M., Togano, T., Takahashi-Niki, K., Lu, J., Honda, A., Taoka, M., et al. (2009). Identification of functional marker proteins in the mammalian growth cone. Proc. Natl. Acad. Sci. U.S.A. 106, 17211-17216. doi: 10.1073/pnas. 0904092106

Oohashi, T., Zhou, X. H., Feng, K., Richter, B., Morgelin, M., Perez, M. T., et al. (1999). Mouse ten-m/Odz is a new family of dimeric type II transmembrane proteins expressed in many tissues. J. Cell Biol. 145, 563-577. doi: 10.1083/jcb. 145.3.563

O’Sullivan, M. L., de Wit, J., Savas, J. N., Comoletti, D., Otto-Hitt, S., Yates, J. R., et al. (2012). FLRT proteins are endogenous latrophilin ligands and regulate excitatory synapse development. Neuron 73, 903-910. doi: 10.1016/j.neuron. 2012.01.018

O'Sullivan, M. L., Martini, F., von Daake, S., Comoletti, D., and Ghosh, A. (2014). LPHN3, a presynaptic adhesion-GPCR implicated in ADHD, regulates the strength of neocortical layer 2/3 synaptic input to layer 5. Neural Dev. 9:7. doi: 10.1186/1749-8104-9-7

Prömel, S., Frickenhaus, M., Hughes, S., Mestek, L., Staunton, D., Woollard, A., et al. (2012). The GPS motif is a molecular switch for bimodal activities of adhesion class G protein-coupled receptors. Cell Rep. 2, 321-331. doi: 10.1016/ j.celrep.2012.06.015

Rubin, B. P., Tucker, R. P., Brown-Luedi, M., Martin, D., and ChiquetEhrismann, R. (2002). Teneurin 2 is expressed by the neurons of the thalamofugal visual system in situ and promotes homophilic cell-cell adhesion in vitro. Development $129,4697-4705$.

Saitou, N., and Nei, M. (1987). The neighbor-joining method: a new method for reconstructing phylogenetic trees. Mol. Biol. Evol. 4, 406-425. doi: 10.1093/ oxfordjournals.molbev.a040454

Schioth, H. B., Nordstrom, K. J., and Fredriksson, R. (2010). The adhesion GPCRs; gene repertoire, phylogeny and evolution. Adv. Exp. Med. Biol. 706, 1-13. doi: 10.1007/978-1-4419-7913-1_1

Scholz, N., Gehring, J., Guan, C., Ljaschenko, D., Fischer, R., Lakshmanan, V., et al. (2015). The adhesion GPCR latrophilin/CIRL shapes mechanosensation. Cell Rep. 11, 866-874. doi: 10.1016/j.celrep.2015.04.008

Scholz, N., Guan, C., Nieberler, M., Grotemeyer, A., Maiellaro, I., Gao, S., et al. (2017). Mechano-dependent signaling by Latrophilin/CIRL quenches cAMP in proprioceptive neurons. eLife 6:e28360. doi: 10.7554/eLife.28360

Silva, J. P., Lelianova, V. G., Ermolyuk, Y. S., Vysokov, N., Hitchen, P. G., Berninghausen, O., et al. (2011). Latrophilin 1 and its endogenous ligand Lasso/teneurin-2 form a high-affinity transsynaptic receptor pair with signaling capabilities. Proc. Natl. Acad. Sci. U.S.A. 108, 12113-12118. doi: 10.1073/pnas. 1019434108

Sugita, S., Ichtchenko, K., Khvotchev, M., and Sudhof, T. C. (1998). alphaLatrotoxin receptor CIRL/latrophilin 1 (CL1) defines an unusual family of ubiquitous G-protein-linked receptors. G-protein coupling not required for triggering exocytosis. J. Biol. Chem. 273, 32715-32724. doi: 10.1074/jbc.273.49. 32715

Tan, L. A., Al Chawaf, A., Vaccarino, F. J., Boutros, P. C., and Lovejoy, D. A. (2011). Teneurin C-terminal associated peptide (TCAP)-1 modulates dendritic morphology in hippocampal neurons and decreases anxiety-like behaviors in rats. Physiol. Behav. 104, 199-204. doi: 10.1016/j.physbeh.2011. 03.015

Tobaben, S., Sudhof, T. C., and Stahl, B. (2000). The G protein-coupled receptor CL1 interacts directly with proteins of the Shank family. J. Biol. Chem. 275, 36204-36210. doi: 10.1074/jbc.M006448200

Tucker, R. P., Beckmann, J., Leachman, N. T., Scholer, J., and ChiquetEhrismann, R. (2012). Phylogenetic analysis of the teneurins: conserved features and premetazoan ancestry. Mol. Biol. Evol. 29, 1019-1029. doi: 10.1093/molbev/ msr271

Tucker, R. P., and Chiquet-Ehrismann, R. (2006). Teneurins: a conserved family of transmembrane proteins involved in intercellular signaling during development. Dev. Biol. 290, 237-245. doi: 10.1016/j.ydbio.2005. 11.038

Tucker, R. P., Martin, D., Kos, R., and Chiquet-Ehrismann, R. (2000). The expression of teneurin-4 in the avian embryo. Mech. Dev. 98, 187-191. doi: 10.1016/S0925-4773(00)00444-5

Vysokov, N. V., Silva, J. P., Lelianova, V. G., Ho, C., Djamgoz, M. B., Tonevitsky, A. G., et al. (2016). The mechanism of regulated release of Lasso/Teneurin-2. Front. Mol. Neurosci. 9:59. doi: 10.3389/fnmol.2016.00059

Vysokov, N. V., Silva, J. P., Lelianova, V. G., Suckling, J., Cassidy, J., Blackburn, J. K., et al. (2018). Proteolytically released Lasso/teneurin-2 induces axonal attraction by interacting with latrophilin-1 on axonal growth cones. eLife 7:e37935. doi: 10.7554/eLife.37935

Wallis, D., Hill, D. S., Mendez, I. A., Abbott, L. C., Finnell, R. H., Wellman, P. J., et al. (2012). Initial characterization of mice null for Lphn3, a gene implicated in ADHD and addiction. Brain Res. 1463, 85-92. doi: 10.1016/j.brainres.2012. 04.053

Wang, L., Rotzinger, S., Al Chawaf, A., Elias, C. F., Barsyte-Lovejoy, D., Qian, X., et al. (2005). Teneurin proteins possess a carboxy terminal sequence with neuromodulatory activity. Brain Res. Mol. Brain Res. 133, 253-265. doi: 10. 1016/j.molbrainres.2004.10.019

Woelfle, R., D'Aquila, A. L., and Lovejoy, D. A. (2016). Teneurins, TCAP, and latrophilins: roles in the etiology of mood disorders. Transl. Neurosci. 7, 17-23. doi: 10.1515/tnsci-2016-0004

Woelfle, R., D’Aquila, A. L., Pavlovic, T., Husic, M., and Lovejoy, D. A. (2015). Ancient interaction between the teneurin C-terminal associated peptides (TCAP) and latrophilin ligand-receptor coupling: a role in behavior. Front. Neurosci. 9:146. doi: 10.3389/fnins.2015.00146

Zhou, X. H., Brandau, O., Feng, K., Oohashi, T., Ninomiya, Y., Rauch, U., et al. (2003). The murine Ten-m/Odz genes show distinct but overlapping expression patterns during development and in adult brain. Gene Expr. Patterns 3, 397405. doi: 10.1016/S1567-133X(03)00087-5

Zuckerkandl, S., and Pauling, L. (1965). "Evolutionary divergence and convergence in proteins," in Evolving Genes and Proteins, eds V. Bryson and H. J. Vogel (New York, NY: Academic Press), 97-166. doi: 10.1016/B978-1-4832-2734-4. 50017-6

Conflict of Interest Statement: The authors declare that the research was conducted in the absence of any commercial or financial relationships that could be construed as a potential conflict of interest.

Copyright (c) 2019 Schöneberg and Prömel. This is an open-access article distributed under the terms of the Creative Commons Attribution License (CC BY). The use, distribution or reproduction in other forums is permitted, provided the original author(s) and the copyright owner(s) are credited and that the original publication in this journal is cited, in accordance with accepted academic practice. No use, distribution or reproduction is permitted which does not comply with these terms. 